\title{
Interoperability Framework for Vehicular Connectivity in Advanced Heterogeneous Vehicular Network
}

\author{
Saied M. Abd El-atty ${ }^{1}$, Konstantinos Lizos \\ Electronics and Electrical Communications Eng. Department, Faculty of Electronic Engineering, \\ Menoufia University, 32952, Menouf, Egypt. \\ Department of Informatics, Faculty of Mathematics and Natural Sciences, University of Oslo, Norway \\ ${ }^{1}$ Arts and Science College, Salman Bin Abdulaziz University, 054-11991 Wadi Adwassir, Riyadh, Saudi Arabia \\ s.soliman@sau.edu.sa, konstantinoslizos@gmail.com
}

\begin{abstract}
Advanced heterogeneous vehicular network (AHVN) is a promising architecture for providing vehicular services in the next generation of vehicular networks. AHVN is an integrated architecture between vehicular ad hoc networks and existing cellular wireless networks. In this work, we propose a Multihop vehicular connectivity model in $\mathrm{V} 2 \mathrm{~V}$ system, which depends on the physical characteristics of the roadways and false hop initiation connectivity. Then, we determine the failure probability of vehicular connectivity in V2V system. Based on interoperability utility, we employ the failure connectivity probability as a handover criterion to communicate with V2R networks. Subsequently, we propose an efficient medium access control (MAC) method based on collaborative codes for resource management in AHVN. As a result, we determine the failure access probability by employing a Markov chain model. The analysis of the proposed MAC in terms of transmission capacity, delay and access failure probability is driven. The numerical and simulation results demonstrate the effectiveness of the proposed framework.
\end{abstract}

Index Terms - Failure Connectivity Probability; Failure Access Probability; MAC; V2V; V2R; AHVN.

\section{INTRODUCTION}

Vehicle to vehicle (V2V) and vehicle to roadside infrastructure (V2R) communications are usually integrated to design a ubiquitous, cooperative communication system, based on the coverage area. $\mathrm{V} 2 \mathrm{~V}$ communications involve vehicular nodes on the road that form a vehicular ad hoc network (VANET). However, V2R involves vehicular nodes, roadside base stations and access points connected with Internet. On the other hand, intelligent transportation systems (ITSs) are poised to play a fundamental rule in introducing the updated and most suitable solutions for safety applications, while non-safety services engage communication in with the commercial wireless networks (GSM, UMTS/HSPA and WiMAX/LTE).
In order to support various safety and non-safety services with the desired quality of service (QoS), vehicles in vehicular networks should not only follow specific protocols, but also cooperate with each other, to enhance the overall network performance and efficiently utilize resources [1] and [2]. Therefore, the idea of combining V2V based systems with the current cellular systems is a feasible solution not only for improving the sustainability of the ITS (particularly for safety related applications), but also for increasing the throughput. The vehicular communications have recently envisioned interests from both academia and industry. The main important cooperation-related parameter between V2R and $\mathrm{V} 2 \mathrm{~V}$ is calculating the parameter of the communication path known as dedicated short range communications (DSRC) cost.

However, DSRC technology will only be effective when it is ubiquitously deployed but this will not happen due to the restrictions of DSRC deployment in passenger's vehicles. On the other hand, IEEE 802.11p standards have issued the wireless access in vehicular environment (WAVE) to support vehicular services [3] and [4]. Most studies in the area of vehicular networks only provide a single type of service in such networks and are only based on a non-cooperative radio access networks. Therefore, the integration of existing wireless access technologies with ITS enables a plethora of services, such as accidents avoidance, emergency calls, traffic congestion control, vehicle entertainment, Internet access and mobile business.

The advanced heterogeneous vehicular network (AHVN) is a promising candidate network for next generation vehicular networks [5]. AHVN deploys multiple radios and multiple access technologies in a cooperative communication's manner and thereby vehicular services can be supported through $\mathrm{V} 2 \mathrm{~V}$ and $\mathrm{V} 2 \mathrm{R}$ integration. $\mathrm{V} 2 \mathrm{R}$ communications involve vehicular nodes and road side base stations. In sequel, each vehicle can send or receive data to/from base station (BS). However, if a vehicle could not directly send its data to BS, it can relay its data to other vehicle until the data reach the BS using Multihop path transmission. In addition, by deploying AHVN, the 
vehicles are able to request social services with different requirements in terms of delay, bandwidth, error rate, coverage area, etc. at any time and any place. Current access technologies such as wireless LANs (WLAN IEEE $802.11 \mathrm{a} / \mathrm{b} / \mathrm{g} / \mathrm{h} / \mathrm{p}$ standards), WiMAX (IEEE 802.16 a/e standards), ultra wideband (UWB IEEE 802.15.3a standard), $3 \mathrm{G}$ and $4 \mathrm{G}$ cellular mobile networks are designed for specific service requirements [5].

Without loss of generality, we deploy a $4 \mathrm{G}$ wireless technology (backward compatibility with $2 \mathrm{G}, 3 \mathrm{G}$ ) for vehicular services that provides a very broad coverage, supports high-mobility vehicles and smoother handover. However, the capacity of BS may be diminished when the code collision happens. Subsequently, the management of the wireless access in AHVN is also a challenge issue. In this study, we firstly propose a Multihop connectivity model in order to determine the failure probability of vehicular connectivity $\left(P_{f}\right)$ in $\mathrm{V} 2 \mathrm{~V}$ system, $P_{f}$ depends on the probability of false hop connectivity, headway distance and received signal strength (RSS). Secondly, based on the interoperability utility, we employ the failure connectivity probability as a handover criterion to communicate with V2R. Next, we propose an efficient medium access control (MAC) collaborative-based codes scheme for resource management in AHVN. Finally, we developed a Markov chain model in order to determine the probability of failure access to V2R system.

The remainder of the paper is organized as follows. The related work is discussed in Section II. A vehicular communications framework in AHVN which includes the interoperability mechanism between V2V and V2R system is presented in Section III. In Section IV, we present the numerical and simulation results that evaluate the performance of the proposed framework. Finally, a brief conclusion of this work is given in Section V. The mathematical notations and their physical meaning that will be used in this work are illustrated in Table 1.
TABLE 1 Mathematical notations and physical meaning

\begin{tabular}{|c|c|}
\hline $\begin{array}{l}\text { Mathematical } \\
\text { notations }\end{array}$ & Physical meaning \\
\hline$\mu$ & Probability of market penetration rate \\
\hline$L, W$ & $\begin{array}{l}\text { Roadway is segmented into microcells with } \\
\text { length }(L) \text { and lane width }(W)\end{array}$ \\
\hline$V_{i}, V_{o}, V_{n}$ & $\begin{array}{l}\text { Current vehicle, old vehicle, new vehicle in V2V } \\
\text { multi-hop system }\end{array}$ \\
\hline$l_{v}, d$ & $\begin{array}{l}\text { Distance headway and a portion of distance } \\
\text { headway at point } P \text {, respectively }\end{array}$ \\
\hline$\tau$ & Broadcast signaling delay time \\
\hline$\lambda_{v}, m_{v}$ & Vehicle arrival rate and number of vehicles \\
\hline$R_{0}$ & Maximum transmission range of each vehicle \\
\hline$P_{c}$ & Probability of vehicular connectivity \\
\hline$P_{\text {false }}$ & False probability of initiation hop \\
\hline$P_{f}$ & Failure probability of vehicular connectivity \\
\hline$\phi, \phi_{t h}, \phi_{\min }$ & $\begin{array}{l}\text { Received signal strength (RSS), threshold of } \phi \text {, } \\
\text { minimum value of } \phi\end{array}$ \\
\hline$T$ & Slot duration of packet transmission \\
\hline$p_{j}$ & $\begin{array}{l}\text { The probability of } j \text { licensed vehicles are active at } \\
\text { a given time slot }\end{array}$ \\
\hline$C$ & The available code in BS \\
\hline$P_{s}$ & $\begin{array}{l}\text { The probability of successful transmission for an } \\
\text { active vehicle }\end{array}$ \\
\hline$I(P)$ & $\begin{array}{l}\text { Denotes to the interoperability function for the } \\
\text { event of failure probability }\left(P_{f}\right) \text { of vehicular } \\
\text { connectivity in V2V }\end{array}$ \\
\hline$\eta$ and $g$ & $\begin{array}{l}\text { The transmission capacity and the gain in } \mathrm{V} 2 \mathrm{R} \\
\text { network respectively. }\end{array}$ \\
\hline$P_{A F}$ & Access failure probability in V2R network. \\
\hline$D$ & $\begin{array}{l}\text { The average delay for successful packet } \\
\text { transmission }\end{array}$ \\
\hline
\end{tabular}

\section{RELATED WORK}

Intelligent transportation systems (ITS) have issued the standards for vehicular communications, according to inter-vehicle communications (IVC) technology. This type of communication is dynamic by nature due to the vehicle mobility and network topology, i.e. called vehicular ad hoc network (VANET) or vehicle to vehicle $\mathrm{V} 2 \mathrm{~V}$, enabling the deployment of Vehicular Social Networks. The connectivity probability in V2V has been studied through many research works by analytical and simulation models [6] and [18]. However, emerging wireless services such as social services in vehicular networks make the medium access of V2V system not suitable to achieve the QoS requirements of such services. Therefore, the integration architecture of V2V and the existing wireless access technology would contribute to better access services and to achieve the QoS requirements. In [5], the authors presented different architectures of advanced heterogeneous vehicular network (AHVN) for vehicular telematics services. They lightened the major challenges and approaches for designing the AHVN such as wireless access strategies, network selection, MAC protocols, etc.

The authors in [7], proposed the basic guidelines design for network integration architecture of $\mathrm{V} 2 \mathrm{~V}$ and $3 \mathrm{G}$ networks. The main purpose of this pivotal research is to select only a minimum number of vehicles to communicate with $3 \mathrm{G}$ network as gateways. In sequel, the authors in [8] introduced integration architecture between $\mathrm{V} 2 \mathrm{~V}$ and $3 \mathrm{G}$ networks by deploying clustering 
gateway. They made use of UMTS received signal strength (RSS) metric of the vehicles instead of vehicle's velocity variations for dynamic clustering mechanism. On the other hand, the authors in [9] developed an analytical model to fully characterize the access probability and connectivity probability performance for infrastructure-based vehicular relay networks. This research is capable of improving access and connectivity probabilities in V2R and V2V based relay network.

Furthermore, in terms of MAC protocols for $\mathrm{V} 2 \mathrm{~V}$ systems, there are many works that have been investigated [10] and [11]. Recently, the authors in [12] proposed a MAC mechanism based on distributed coordination function (DCF) for V2R communication. They developed an analytical model to evaluate the performance of DCF in high-speed vehicular environment. Consequently, the authors in [13] proposed a contextual cooperative congestion control policy between vehicles and infrastructure nodes that exploits the traffic information of each vehicle to reduce the unnecessary interferences. In addition, the authors in [14] proposed a novel adaptive distributed cooperative relaying MAC protocol for vehicular networks, this research addressed the inherent problems of MAC based IEEE 802.11 standard.

As a popular and emerging wireless standard, the Vehicular Social Networks (VSNs) present a qualified framework, for the formation of trust-oriented communities among moving vehicles. Since this technology is fairly new, the related work is scarce and inadequate but with great interest and impact for urban environments. In [21], a model is presented to cope with the administrative-related issues through a dynamic trust management module. This model is analyzed mathematically to assess the probability of deceitful penetration (Node-to-Node, Node-to-Group) while exhibiting a low-latency ratio Latency is measured by the level of interaction and the responses that occurred.

Resource allocation in VSNs requires sensitive balancing, since it mitigates the complexity of wireless networks in decentralized vehicle structures with potentially heterogeneous diversified traffic requirements. More specifically, in [23] the challenges of VSNs in this context are summarized:

- Effortless human intervention (by utilizing Human-Machine Interface (HMI) and command execution through speech channels)

- Adaptation/penetration of supported capabilities over diversified hardware and application heterogeneous devices

- Anonymity and privacy

- Interoperability in reference to collecting and administrating information

- Efficient use of network resources

- Authentication / Security related issues

Privacy and security in particular are extensively covered in [24] for social-aware vehicle communication.
Although the scenario is both feasible and successful, the presence of trusted super-entity, centralizing audit mechanisms, is essential. In [22], a scheduling policy is introduced and analyzed to effectively integrate the unremitting issues of social needs into lower network modules. The authors examine the Centrality Degree i.e. an entity is more central than others if it is more visible /prominent to other entities. Moreover, the proposed scheduling solution encompasses two additional factors: the level of service request from each node and the separate service types that request is comprised of to derive a scheduling decision.

To the best of our knowledge, no research discussed the failure connectivity probability (Pf) in V2V system that is caused by different parameters such as the physical characteristics of the roadways, vehicular traffic density, broadcast signaling delay, false hop initiation connectivity. Moreover, the interoperability mechanism for employing the failure connectivity probability as handover criterion for establishing a communication link to V2R is proposed.

\section{FRAMEWORK FOR VEHICULAR CONNECTIVITY IN AHVN}

The framework for vehicular connectivity in AHVN is mainly based on the interoperability between $\mathrm{V} 2 \mathrm{~V}$ and V2R networks. A simplified architecture for the AHVN is illustrated in Fig.1.

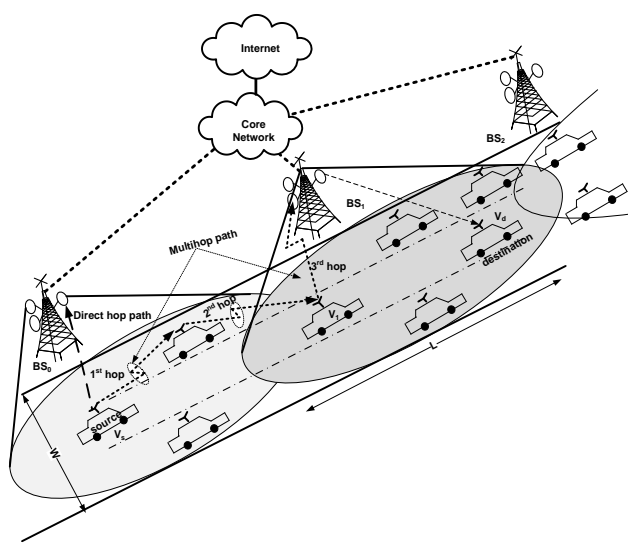

Figure 1: A simplified architecture of AHVN

The basic components of the network infrastructure are V2V system based-Multihop strategy, BS, core mobile networks and Internet. The deployment of AHVN enables vehicles to get access to BS outside their transmission range via transmission strategies, single hop or Multihop transmission, which improves the network coverage [5]. Further, cooperative use of the multiple radios enhances the performance of the networks by allowing more parallel transmissions in different frequencies. The connectivity over V2V networks depends on inter-vehicle connectivity (IVC) basis and Multihop transmission strategy. In addition, the vehicle can directly connect through a single hop path as long as its location inside the coverage area of the serving BS. On the other hand, the vehicle can use a 
V2V Multihop path connectivity to communicate with BS during its movement from one cell segment to other [15].

\section{A. Interoperability utility between $V 2 V$ and $V 2 R$ systems}

In order to provide interoperability communication between V2V with V2R systems, two important concerns need to be effectively addressed. This functionality could be accomplished with the ability of vehicle to select the appropriate access network during its roaming from one cell to the other. Firstly, the vehicle may establish a Multihop connectivity link by using DSRC technology in V2V network according to physical characteristics of roadway such as headway distance, vehicular traffic density, hop false initiation connectivity and received signal strength (RSS).

However, this connectivity may fail due to unavailability of physical link in $\mathrm{V} 2 \mathrm{~V}$ network; hence the probability of failure connectivity (Pf) in $\mathrm{V} 2 \mathrm{~V}$ network should be estimated. The next claim, the received signal strength (RSS) at vehicle will be deteriorated; thereby we make use of the interoperability utility and the probability of failure connectivity $(\mathrm{Pf})$ in order to access collaborative codes in roadside infrastructure (V2R) to get the vehicular services or Internet services. Therefore, we compute the successful transmission probability in $\mathrm{V} 2 \mathrm{R}$ network taking into account the probability of failure connectivity $(\mathrm{Pf})$ in V2V network. The algorithm of interoperability communications between V2V and V2R systems can be concluded as follows:

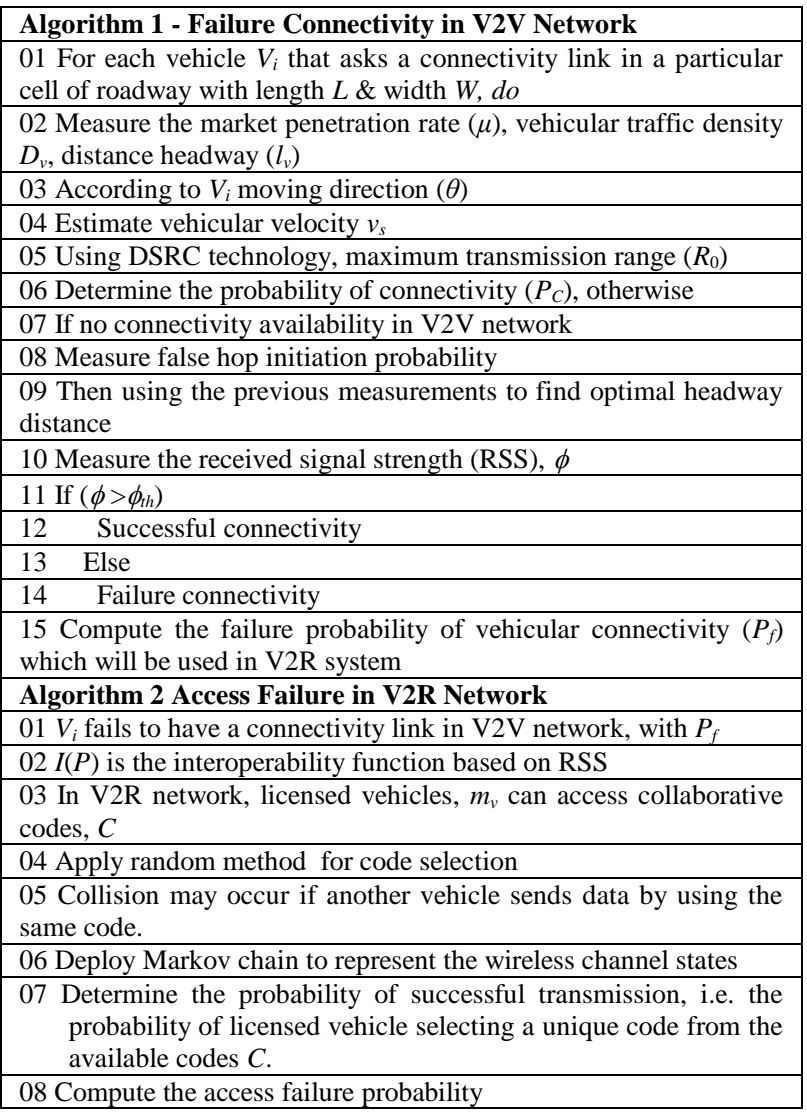

\section{B. V2V system}

\section{1) Mobility model}

In inter-vehicle connectivity (IVC) systems, vehicles equipped with wireless communication devices along roadways establish V2V system [16]. The feasible connectivity depends on the vehicular traffic density on the roadway. The probability of whether or not a vehicle equipped with wireless communication devices is random with the probability of market penetration rate, denoted by $\mu$ or $(1-\mu)$ respectively. We consider the roadway, segmented into microcells with $n$ up and $n$ down lanes, and we assume each lane with length $\mathrm{L}$ and width $\mathrm{W}$, as shown in Fig.2. The movement of the vehicles in each lane is considered to be in the same direction. We consider the scenario in which a vehicle (Vi) is currently connected by old vehicle (Vo). $\mathrm{Vi}$ is moving with a space mean velocity vs. Let vs be uniformly distributed in [vmin, vmax], therefore the probability density function (pdf) of vs can be expressed as follows:

$f_{v}\left(v_{s}\right)=\frac{1}{v_{\max }-v_{\min }}, \quad v_{\max }<v_{s}<v_{\min }$

There is always a relationship between the mobility of vehicles and the network connectivity of the ad hoc system. For example, during its course of movement in a typical lane, vehicle Vi is connected with old vehicle Vo and hops a new communication path with new vehicle $\mathrm{Vn}$

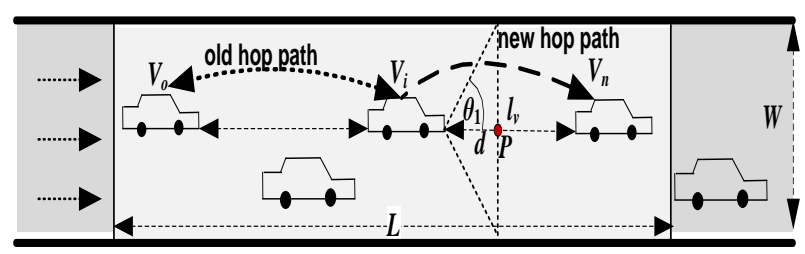

Figure 2: A segment of highway microcells structure.

Distance headway (lv) is a key parameter in calculating the overall capacity of vehicular ad hoc network [17]. Therefore, distance headway is related to vehicular traffic density (Dv) as follows $1 / \mathrm{Dv}$. The relationship between the volume of vehicular traffic $\mathrm{Qv}$ and density is given by $\mathrm{Qv}=\mathrm{Dv} \times \mathrm{vs}$, where vs is a function of the vehicular velocity through the following relation, vs $=$ vf $[1-\mathrm{Dv} / \mathrm{Djam}]$, where vf is the mean velocity for free flow conditions and Djam is the density under jam traffic.

Under free flowing, all vehicles are able to move at the velocity characteristics of the roadway, as determined by its physical features, velocity limits, traffic lights, etc. When vehicle $\mathrm{Vi}$ reaches the point $\mathrm{P}$ at distance (d) of the distance headway (lv) then it probably hops with the new Vn, during the desirable distance headway before being out of transmission range. When $\mathrm{Vi}$ is located at point $\mathrm{P}$, we assume that it can 
move in any direction with equal probability, therefore the pdf of Vi's direction of motion $\theta$ can be expressed as follows:

$$
f_{\theta}(\theta)=\frac{1}{2 \pi}, \quad-\pi<\theta<\pi
$$

Without loss of generality, we consider the direction and velocity of $\mathrm{Vi}$ remaining the same from point $\mathrm{P}$ till it moves out of the coverage area of new vehicle $\mathrm{Vn}$. As the distance headway to new vehicle is small, this assumption is realistic. For example, for a vehicle moving at $100 \mathrm{~km} / \mathrm{hr}$ considering the broadcast signaling delay time $(\tau)$ is $2 \mathrm{sec}$, d will be $50 \mathrm{~m}$. Hence during high vehicular traffic density, the velocity and also the broadcast signaling delay will be decreased; therefore $\mathrm{d}$ will be much smaller.

\section{2) Vehicular connectivity}

In a free-flow traffic highway, we are interested in those vehicles that fall in the interval $[0, \mathrm{~L}]$ as illustrated in Fig.II, the number of vehicles at time $t$ is a random variable. Let $\operatorname{Mv}(\mathrm{t})$ denote to the number of vehicles in $[0, \mathrm{~L}]$ at time $\mathrm{t}$, and $\mathrm{K}(\mathrm{t})$ denote the total number of vehicles that arrive at the time interval $[0, \mathrm{t}]$. Let the vehicle arrival rate be $\lambda \mathrm{v}$, then we have:

$$
P\{K(t)=k\}=\frac{\left(\lambda_{v} t\right)^{k}}{k !} e^{-\lambda_{v} t}
$$

The distance headway between two consecutive vehicles is exponentially distributed, i.e., $l v$ is exponentially distributed with the following cumulative distribution function as follows [17]:

$$
F_{c d f}\left(l_{v}\right)=1-e^{-l_{v} \cdot D_{v}}=1-e^{-m_{v} \cdot\left(l_{v} / L\right)}
$$

The distribution probability of $\mathrm{Mv}(\mathrm{t})$ can be derived as follows:

$$
P\left\{M_{v}(t)=m_{v}\right\}=\frac{\left(L \lambda_{v} / v_{s}\right)^{m_{v}}}{m_{v} !} e^{-L \lambda_{v} / v_{s}}
$$

Thereby, $\operatorname{Mv}(\mathrm{t})$ is Poisson distributed with mean $\lambda=\mathrm{L} \lambda \mathrm{v} / \mathrm{vs}$. This is very important for the system designer in order to expect the connectivity probability according to vehicle distribution as well as transmission range. Accordingly, assuming an ad hoc network with mv vehicles Poisson distributed over a segment with length $\mathrm{L}$ and maximum transmissions range of each vehicle $\mathrm{R} 0$. The hop path between two vehicles can be established if distance headway is equal to or less than R0. Then the probability that all $\mathrm{mv}$ vehicles are connected can be expressed as follows [17]:

$$
P_{c}=\operatorname{Pr}\left\{l_{1} \leq R_{0}, l_{2} \leq R_{0}, \ldots, l_{m_{v}-1} \leq R_{0}\right\}
$$

In reality the distance headway li is an independent and identically distributed (iid) random variable, therefore from (4) and (6), Pc is given by

$P_{c}=\prod_{i=1}^{m_{v}-1} \operatorname{Pr}\left\{l_{i} \leq R_{0}\right\}=\left(1-e^{-D_{v} \cdot R_{0}}\right)^{m_{v}-1}$

\section{3) The failure probability of vehicular connectivity}

A successful communication between vehicles is established when a broadcast signaling for initiation connectivity is received before a complete connectivity is executed. As shown in Fig.2, it is illustrated that the initiation of hop communication to the new vehicle (Vn) arises only if Vi's direction of motion from $\mathrm{P}$ within the range $\{-\theta 1, \theta 1\}$, where $\theta 1=\arctan (W / 2 d)$. Otherwise, the false hop initiation probability is a false one. Therefore, by using (2), the false hop initiation probability can be expressed as follows:

$P_{\text {false }}=\int_{-\theta_{1}}^{\theta_{1}} f_{\theta}(\theta) d \theta=1-\frac{1}{\pi} \arctan \left(\frac{W}{2 d}\right)$

The moving direction of $\mathrm{Vi}$ from $\mathrm{P}$ is $\beta \in\{-\theta 1, \theta 1\}$, the time $t$ it takes to move out of the coverage area of the old vehicle (Vo), therefore, the pdf of $t$ can be derived as follows:

$f_{t}(t)= \begin{cases}\frac{d}{\theta_{1} t \sqrt{\left(v_{s} t\right)^{2}}}, & \frac{d}{v_{s}}<t<\frac{d}{\theta_{1} t \sqrt{\left(v_{s} t\right)^{2}}} \\ 0 & \text { otherwise }\end{cases}$

The failure probability of vehicular connectivity, Pf is expressed as:

$$
P_{f}= \begin{cases}1, & \tau>\frac{\sqrt{\left(\frac{W}{2}\right)^{2}+d^{2}}}{v_{s}} \\ p(t<\tau), & \frac{d}{v_{s}}<\tau<\frac{\sqrt{\left(\frac{W}{2}\right)^{2}+d^{2}}}{v_{s}} \\ 0 & \frac{d}{v_{s}} \geq \tau\end{cases}
$$

where $\tau$ is the time delay of signalling broadcast for connectivity and $\mathrm{p}(\mathrm{t}<\tau)$ is the probability that $\mathrm{t}<\tau$ when $\frac{d}{v_{s}}<\tau<\frac{\sqrt{\left(\frac{W}{2}\right)^{2}+d^{2}}}{v_{s}} \quad$, using (9) in order to obtain $\mathrm{p}(\mathrm{t}<\tau)$ as follows:

$$
p(t<\tau)=\int_{\frac{d}{v_{s}}}^{\tau} \frac{d}{\pi t \sqrt{\left(v_{s} t\right)^{2}-d^{2}}} d t \cong \frac{1}{\theta} \arccos \left(\frac{d}{v_{s} \tau}\right)
$$


$P_{f}= \begin{cases}1, & \tau>\frac{\sqrt{\left(\frac{W}{2}\right)^{2}+d^{2}}}{v_{s}} \\ \frac{1}{\theta} \arccos \left(\frac{d}{v_{s} \tau}\right), & \frac{d}{v_{s}}<\tau<\frac{\sqrt{\left(\frac{W}{2}\right)^{2}+d^{2}}}{v_{s}} \\ 0 & \frac{d}{v_{s}} \geq \tau\end{cases}$

\section{4) Outage probability}

The message hops for one vehicle to next vehicle within the same driving direction [17]. According to the number of the vehicles equipped with wireless device, that is the market penetration rate $(\mu)$, therefore if there is a receiver vehicle in the broadcast range of sending vehicle, a message hopping is possible. For successful message hopping, the distance headway must be smaller than the maximum transmission range; otherwise, the message hopping fails. The probability of finding a receiver vehicle for message hopping is based on the maximum transmission range (R0) is given by

$$
p\left(l_{v}<R_{0}\right)=\int_{0}^{R_{0}} e^{-l_{v}\left(\mu \cdot D_{v}\right)} d l_{v}=1-e^{-R_{0}\left(\mu \cdot D_{v}\right)}
$$

Therefore, the connectivity probability over a distance (for example, $\mathrm{r}=1 \mathrm{~km}$ ) in one directional roadway is based on the successful probability of message hopping in IVC systems and it is given by [10]:

$$
\begin{aligned}
& P_{c}(r)=1-\sum_{i=1}^{\frac{r}{R_{0}} \mid} \frac{\left(-\mu \cdot D_{v}\right)^{i-1}}{(i-1) !}\left(r-i R_{0}\right)^{i-1} \\
& \times\left[1+\frac{\left(\mu \cdot D_{v}\right)}{i}\left(r-i R_{0}\right)\right] e^{-i R_{0}\left(\mu \cdot D_{v}\right)}
\end{aligned}
$$

where $\lfloor r / R 0\rfloor$ is the largest integer smaller than or equal to $\mathrm{r} / \mathrm{R} 0$. Therefore, the outage connectivity probability (Poutage) can be defined as the event that there is at least one vehicle disconnected from the remaining of the Multihop vehicles in the network. Hence Poutage can be expressed as follows:

$$
P_{\text {outage }}(r)=1-P_{c}(r)
$$

\section{5) Optimal headway distance}

The hop initiation for connectivity with the new vehicle $\mathrm{Vn}$ is based on the received signal strength $(\phi)$. In sequel $\phi$ depends on the optimal distance for successful connectivity. Let the threshold of received signal strength is $\phi$ th and the minimum value of received signal strength is $\phi \mathrm{min}$, required for successful connectivity between Vi and Vn. The optimal value of $\mathrm{d}$ can be estimated for a desired value of Pf. Equation (12) is a nonlinear equation of $\mathrm{d}$. A closed form expression may not be always possible. However, an approximate value of $d$ can be estimated using:

$$
P_{f} \cong \frac{\cos ^{-1}\left(\frac{d}{v_{s} \tau}\right)}{\tan ^{-1}\left(\frac{W}{2 d}\right)}=\frac{\frac{\pi}{2}-\frac{d}{v_{s} \tau}}{\frac{\pi}{2}-\frac{2 d}{\sqrt{(2 d)^{2}+W^{2}}}}
$$

Numerical methods can be used to determine d. We employ the Bisection numerical method to solve for $\mathrm{d}$. Hence, calculation of $d$ does not have much computational complexity and can be easily implemented in the vehicle's radio software. Fig. 3 shows the numerical result for solution Eq.(16), the relationship between the optimal headway distance and the failure probability of connectivity at different values of $\tau$ when velocity is vs $=80 \mathrm{~km} / \mathrm{hr}$. For example, as illustrated in the Fig. 3 at $\tau=3 \mathrm{sec}$ and a threshold value of $\mathrm{Pf}=0.4$, the optimal headway distance for successful connectivity in $\mathrm{V} 2 \mathrm{~V}$ is approximately less than or equal to $60 \mathrm{~m}$.

Once the optimal distance (d0) is determined, the corresponding value of RSS is calculated using the path loss model as follows [19]:

$$
P_{r}(l)=P_{r}\left(d_{0}\right) \cdot\left(\frac{d_{0}}{l}\right)^{\alpha}+\sigma
$$

where 1 is the distance between $\operatorname{Vi} \& \operatorname{Vn}$ and $\operatorname{Pr}(\mathrm{d} 0)$ is the received power at a known reference distance $\mathrm{d} 0$, it can be obtained for line of sight (LOS) or non-line of sight (NLOS) vehicular environment. $\alpha$ is the path loss exponent, its typical value ranges from 2 to 8 in a vehicular environment [19]. Finally $\sigma$ is zero mean Gaussian random variable. The typical value of $\sigma$ is $8 \mathrm{~dB}$. The value of $\sigma$ depends on the lane size $(\mathrm{W})$. Therefore, received signal strength $(\phi)$ value when $\mathrm{Vi}$ at a $\mathrm{d}$ distance from $\mathrm{Vn}$ is given by

$$
\phi=10 \log _{10}\left[P_{r}(W-d)\right]
$$

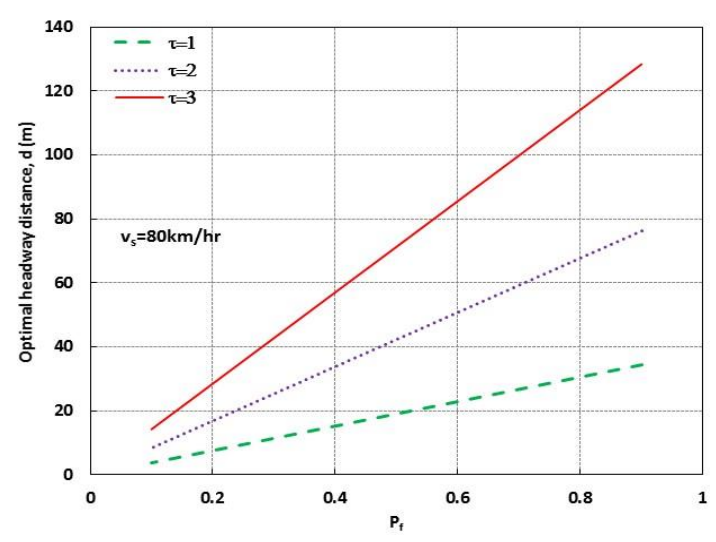

Figure 3: Optimal headway distance versus Pf at different values of $\tau$. 


\section{Vehicular to roadside infrastructure (V2R) model}

\section{1) Collaborative codes}

In each $\mathrm{BS}$, we assume that there are $\mathrm{C}$ collaborative codes for exclusively use for mv licensed vehicles (where $\mathrm{C}<\mathrm{mv}$ ). We consider mv vehicles using the same coding technique but at different time slots. When the failure probability $(\mathrm{Pf})$ of vehicular connectivity in $\mathrm{V} 2 \mathrm{~V}$ increased above a threshold value, a licensed vehicle is able to communicate with the BS for code access by using Multihop transmission strategy. A licensed vehicle may randomly select one of the available collaborative codes $(\mathrm{C})$ and sends its data traffic. A maximum of $\mathrm{C}$ vehicles can utilize the channel simultaneously, if they have used different codes, otherwise collision may occur.

\section{2) MAC based collaborative codes}

In vehicular to roadside infrastructure (V2R) communications, MAC protocol defines how the vehicles and the base station (BS) share common radio codes for vehicular communications. On the other hand, in $\mathrm{V} 2 \mathrm{~V}$ communication, MAC protocol defines how the vehicular nodes share codes to transmit data among them [5]. However, due to the vehicle's mobility frequent handover and the high variant of topology along the road; an efficient MAC protocol is a challenging issue. Therefore, the integrated architecture of AHVN enables vehicles to get access to the cellular system (BS) outside their transmission range via Multihop transmission strategy. In this section, we propose a medium access scheme based-collaborative code for the resource management in AHVN. This scheme favors the vehicles that failed to have a communication link in $\mathrm{V} 2 \mathrm{~V}$; they will have the ability to access the V2R network.

We consider all licensed vehicles to have equal priority and they are able to transmit packets with equal length at any time they want. We assume that the slot duration of packet transmission is denoted by a constant value of $\mathrm{T}$. The probability that a vehicle sends a packet in one slot isu. In any cell segment, the probability of $\mathrm{j}$ licensed vehicles, being active at a given time slot can be expressed as follows:

$p_{j}=\left(\begin{array}{c}m_{v} \\ j\end{array}\right) v^{j}[1-v]^{m_{v}-j}$

When licensed vehicles are active, the probability of successful transmission $s(j)$ is the probability of each vehicle selecting a unique code from the available code $\mathrm{C}$, is given by

$$
s(j)=\left\{\begin{array}{lc}
1, & j=1 \\
\frac{C !}{(C-j)} \times \gamma^{j}, & 1<j \leq C \\
0 & j \geq C
\end{array}\right.
$$

where $\quad=1 / \mathrm{C}$ is the probability that an active vehicle selects a certain code. The probability of successful transmission, Ps for any number of active vehicles is then given by:

$P_{s}=\sum_{j=0}^{C} s(j) \cdot p_{j}$

Based on the above assumptions, the wireless channel can be in one of three states: VANET fail, collided or sending. In our analysis, we utilize Markov chain to represent the wireless channel states of vehicles as shown in Fig.4. State 1 represents the wireless channel status with failure connectivity probability (Pf) in V2V system, with probability $\mathrm{I}(\mathrm{P})$. State 2 describes the collision status with probability [1-Ps-I(P)]. Finally, state 3 represents the sending mode with probability Ps.

According to the Markov chain illustrated in Fig.4, we can obtain the state transition matrix as

$\mathrm{P}=\left[\begin{array}{ccc}I(P) & I(P) & I(P) \\ 1-I(P)-P_{s} & 1-I(P)-P_{s} & 1-I(P)-P_{s} \\ P_{s} & P_{s} & P_{s}\end{array}\right]$

where $\mathrm{I}(\mathrm{P})$ denotes to the interoperability utility for the event of failure probability (Pf) of vehicular connectivity in $\mathrm{V} 2 \mathrm{~V}$ system, i.e. $\mathrm{I}(\mathrm{P})$ is a function of received signal strength $(\phi)$, it is given by:

$I(P)= \begin{cases}P_{f} & \phi<\phi_{t h} \\ p_{0} & \phi \geq \phi_{t h}\end{cases}$

where p0 is the state probability of idle channel and the equilibrium distribution vector $(\Delta)$ can be derived as follows:

$\Delta=\left[\begin{array}{c}I(P) \\ 1-I(P)-P_{s} \\ P_{s}\end{array}\right]$

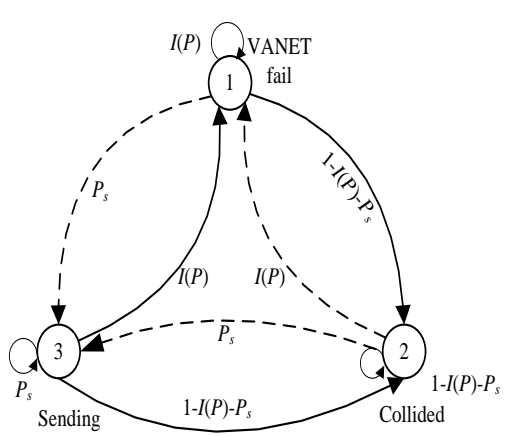

Figure 4: Markov chain of vehicular wireless code states

The transmission capacity $(\eta)$ of the V2R network is given by 
$\eta=\left(\Delta_{1}+\Delta_{2}+\Delta_{3}\right) \sum_{j=1}^{C} j \cdot s(j) \cdot p_{j}$

Additionally, the gain (g) and access failure probability (PAF) in V2R network respectively can be measured by taking a SALOHA as reference basis, in the following manner:

$$
\begin{aligned}
& g=20 \log _{10}\left(\frac{\eta}{\eta_{S A L O H A}}\right) \\
& P_{A F}=1-\frac{\eta}{C \cdot v}
\end{aligned}
$$

where $\frac{\eta}{C \cdot v}$ is the probability of a successful transmission for a given licensed vehicle. On the other hand, the efficiency of the MAC $(\varepsilon)$ can be determined as follows:

$$
\varepsilon=\frac{\eta}{\text { averageinput traffic }}=\frac{\eta}{m_{v} v}
$$

Also, we consider the average duration of the random wait period to be $\tau p$, hence the average delay for successful packet transmission can be expressed as follows:

$$
D=\frac{\tau_{p} \times m_{v} v}{\eta}
$$

\section{NUMERICAL AND SIMULATION RESULTS}

The performance evaluation of the proposed framework is evaluated for a highway as a case study. Thereby, we firstly study the performance of the V2V system, then, according to the interoperability algorithm, we can obtain the behavior of Pf to study the performance of the V2R system.

\section{A. Performance Evaluation in V2V system}

In this section, we study the performance of the $\mathrm{V} 2 \mathrm{~V}$ system model in terms of false initiation probability, connectivity probability and failure connectivity probability. The characteristics of vehicular environment such as Dv, $\mu$, vs and R0 are set to default parameters. A vehicular traffic density in a cell segment $(\mathrm{L}=1 \mathrm{~km})$ of highway is given by $(\mathrm{Dv}=\mathrm{mv} / \mathrm{L})$. The numerical results of the V2V system model are shown in the following figures.
Fig.5 illustrates the probability of Multihop connectivity for increasing Dv when $\mu$ set to 10 per cent at different values of R0. As expected, the connectivity probabilities at high transmission range are higher than the connectivity at low transmission range. The importance of this curve lies in the fact that the designer of Multihop connectivity in vehicular networks has the capability to precisely pre-assess the number of vehicles per highway segment length for a desired level of connectivity.

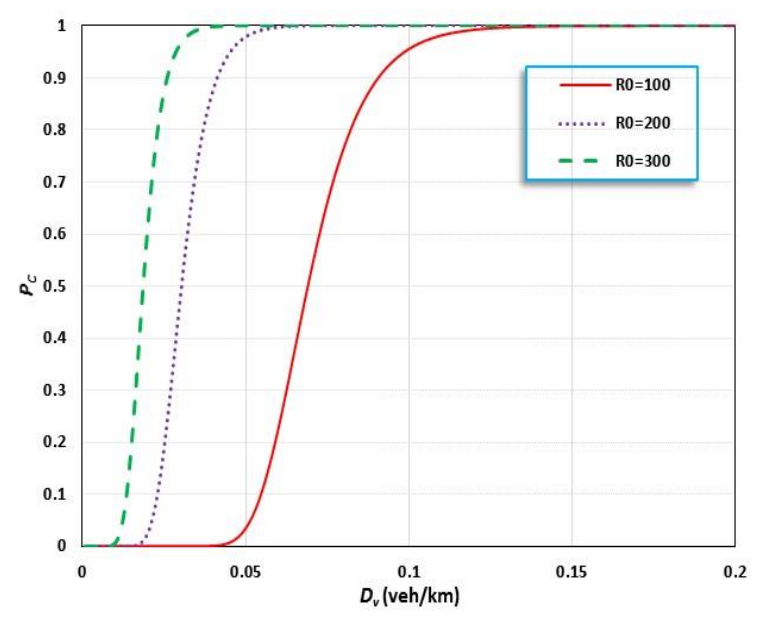

Figure 5: Multi-hop connectivity probability versus vehicular traffic density

Due to the physical characteristics of the highway, the association between probability of false hop initiation connectivity and the portion of distance headway (d) for different lane sizes, W is shown in Fig.6. As we can see, Pfalse increases when the distance headway increases at different values of lane sizes. In addition for each particular value of $\mathrm{W}$, Pfalse is significantly reduced at large values of $\mathrm{W}$. This stems from the fact that the number of vehicles, occupying those lanes has probably elevated. Notably, it shows that the problem of false initiation connectivity becomes more and more severe when lanes size decreases.

Fig.7 shows a correlation between the failure connectivity probability (Pf) and mean space vehicular velocity (vs) for different values of $d$ when $\tau=3 \mathrm{sec}$. We can see that as vs increases, Pf significantly increases. This figure also shows that for a large value of $d$ (i.e., distance headway is small), Pf decreased. In other words, when the vehicular traffic density is high, then the distance headway is small; in sequel Pf is significantly reduced. Obviously, the higher the number of equipped vehicles $\mathrm{V} 2 \mathrm{~V}$ on the highway sector is, the higher the occurrence of having a Multihop connected V2V networks. 


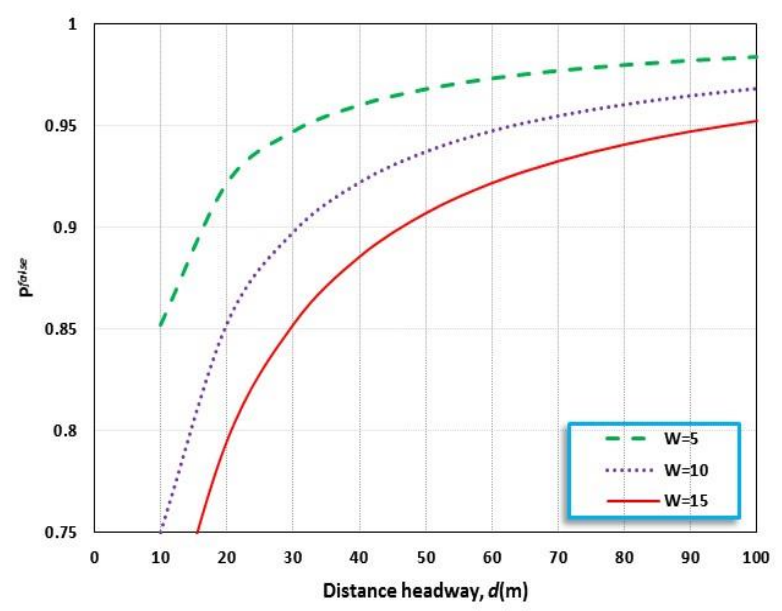

Figure 6: False initiation probability versus distance headway

The successful message hopping probability between the equipped vehicles through IVCs is not only based on the maximum transmission range but also on the vehicular traffic density. The outage connectivity probability versus number of vehicles in a sector $L=1 \mathrm{~km}$ with $\mu$ set to 25 per cent, at different values of $\mathrm{R} 0$ is illustrated at Fig.8. As we can see in this figure, the outage connectivity probability is significantly diminished by increasing the number of vehicles in the highway sector. Furthermore, the outage connectivity probability becomes more and more reduced at high value of maximum transmission range.

\section{B. Performance Evaluation in V2V system}

At BS, we assume that the number of collaborative codes (C) is 2,3 , and 5 codes for exclusive use by licensed vehicles. In addition, based on the interoperability algorithm, the handover criterion to V2R is introduced. In the following numerical results, we adopt the failure probability of connectivity in $\mathrm{V} 2 \mathrm{~V}$ system set to 0.4 as threshold value for handover criterion to V2R system.

Further, we develop a simulation model according to the main parameters of ns-2 simulator for vehicular networks [20]. The simulation scenario is used to keep the tractability of the analytical model. We considered a continuous highway of length $10 \mathrm{~km}$ with 10 cells of length $1 \mathrm{~km}$. Vehicles are generated following a Poisson process with arrival rate 0.3 and $0.5 \mathrm{veh} / \mathrm{sec}$ and the vehicle speed is uniformly distributed with $\{20$, $120 \mathrm{~km} / \mathrm{hr}$. We assume that the number of vehicles per cell $(\mathrm{mv})$ is 50 .

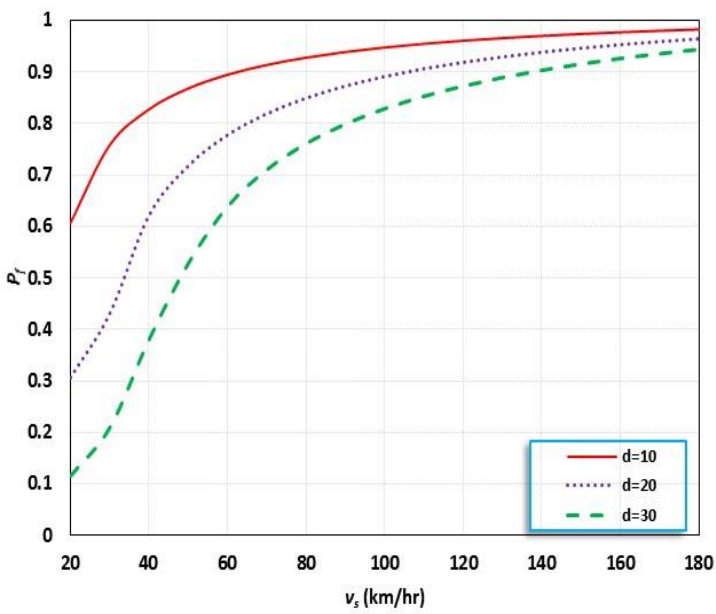

Figure 7: Failure connectivity probability versus vehicular velocity

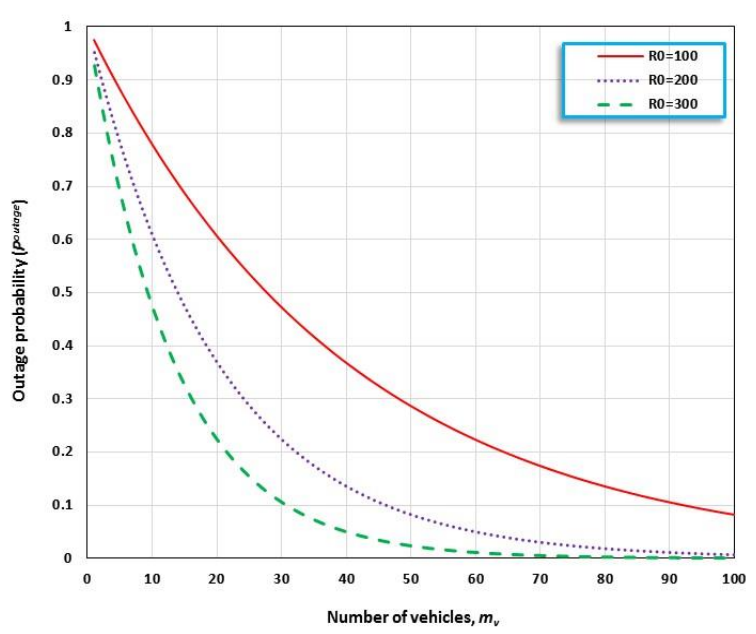

Figure 8: Outage connectivity probability versus vehicles number in sector $\mathrm{L}=1 \mathrm{~km}$

In order to simplify the studying of the performance MAC scheme, we apply a real-time social traffic flows such as voice chat with the following assumptions:

1. The system supports $m_{v}=50$ vehicles.

2. Voice data rate is $64 \mathrm{kbps}$ during activity period.

3. The payload of voice packet is 160 bits/packet; hence voice packet rate is 400packet/sec.

4. The total packet size is 200 bits including the header, FEC encoding, and payload.

5. The wireless code transmission rate is $10 \mathrm{Mbps}$, thus packet transmission delay is $0.02 \mathrm{~ms}$.

6. Maximum threshold packet delay is $3 \mathrm{msec}$. 
Therefore, from the above assumptions, we consider a system with time slot $\mathrm{T}=0.02 \mathrm{~ms}$, $\tau \mathrm{p}$ is equal to one time slot and the average input traffic is expressed by mvv . We now present a snapshot of our simulation model supported by numerical results in order to evaluate the proposed framework in AHVN.

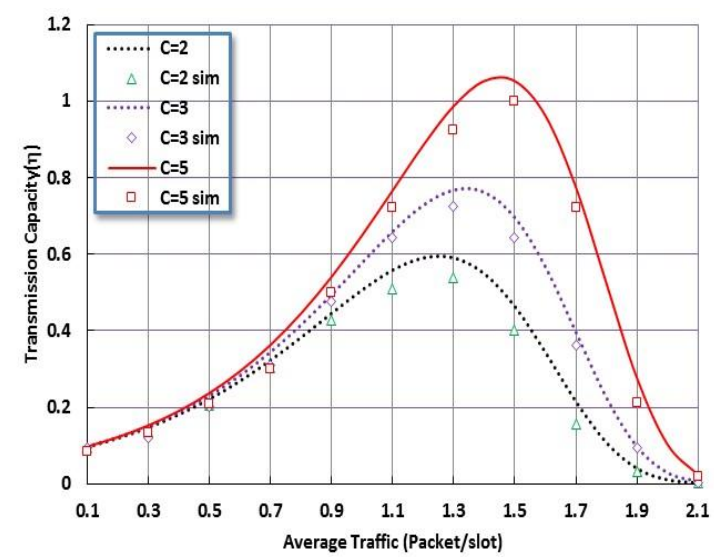

Figure 9: Transmission capacity vs. the average input traffic

Fig.9 shows the transmission capacity ( $\eta$ ) performance as the average input traffic increases. We can see in this plot, we can observe that see the curve of the transmission capacity is getting better when the number of collaborative codes increases; because when the number of the collaborative codes increased, the vehicle's successful transmission probability increases. Furthermore, it is clear that our analysis based Markov chain can evaluate precisely the performance of the proposed MAC. The behavior of the curve indicates that the transmission capacity is steadily increased from low to medium traffic load and the transmission capacity goes down at high traffic load.

Fig.10 shows also the performance of average packet delay at different values of $\mathrm{C}$ by increasing the average input traffic. As we can see, the average packet delay is significantly reduced when the number of the collaborative codes is high. Subsequently, the higher the number of $\mathrm{C}$, the higher the gain of system is, as shown in Fig. 11.

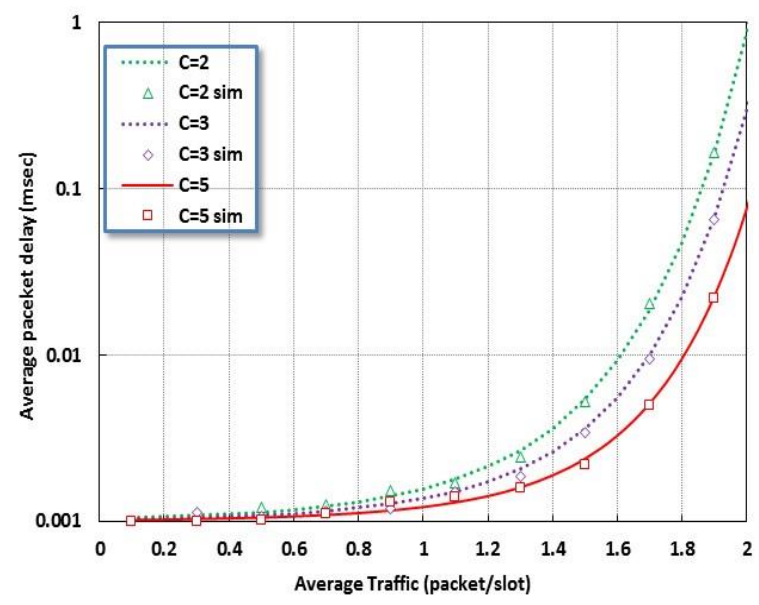

Figure 10: Average packet delay vs. the average input traffic

\section{Performance comparison}

In this section, we introduce the validation of the proposed scheme. We compare the performance of the proposed scheme with adaptive distributed cooperative medium access control (ADC-MAC) [14] and the IEEE 802.11 protocol [18]. ADM-MAC is accomplished through adaptively selecting the most suitable relay or helper and the transmission mode in accordance with the code quality and location of the relay vehicle. In addition, ADM-MAC is also compatible with the standard IEEE 802.11 protocol that depends on the Distributed Coordination Function (DCF) protocol. We adopt our system model to perform the system analysis of the ADM-MAC and IEEE 802.11 protocols.

We observe that by increasing the input average traffic in our system model results in packet size expansion in ADM-MAC scheme and IEEE 802.11 protocol. Fig.12 shows the transmission capacity performance comparison between our scheme and ADM-MAC / IEEE 802.11 schemes. As shown in this figure, it is straightforward that the behavior of the proposed scheme is comparable with both ADM-MAC and IEEE 802.11 protocols. In addition, the transmission capacity of the proposed scheme outperforms both ADM-MAC and IEEE 802.11 schemes.

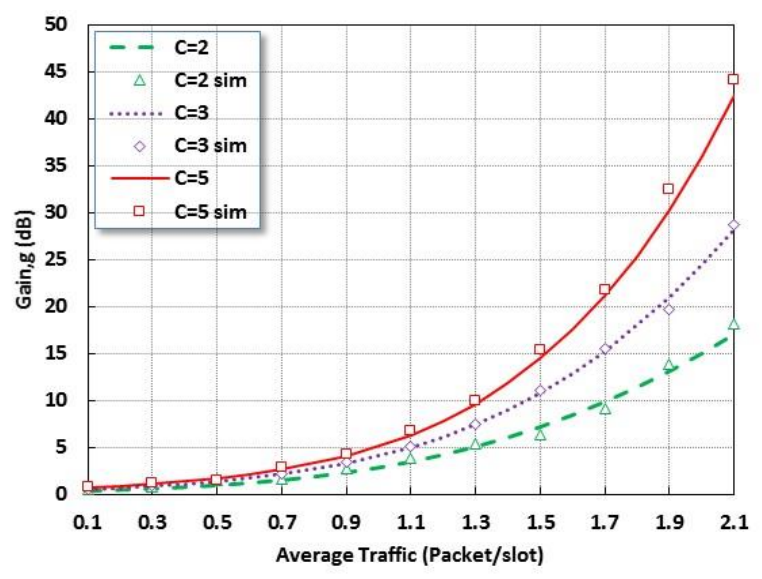

Figure 11: Gain versus the average input traffic

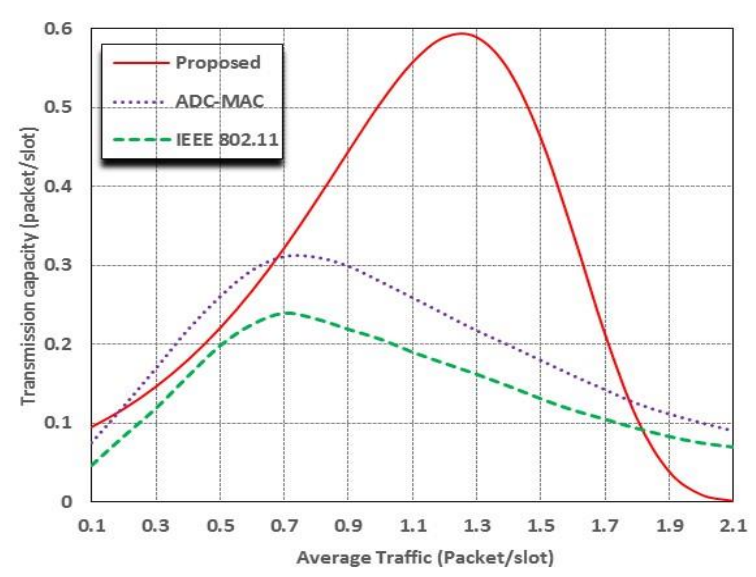

Figure 12: Transmission capacity vs. the average input traffic. 
To summarize, our framework analysis shows that, the vehicular connectivity can be improved by simple integration architecture between V2V and V2R networks as a simplified form of AHVN. As shown in the outcomes, the AHVN network transmission capacity is increased by increasing the number of collaborative code in V2R. Subsequently, access failure and average delay are significantly decreased.

\section{CONCLUSIONS AND FUTURE WORK}

We presented a framework for emerging vehicular service in advanced heterogeneous vehicular network $(\mathrm{AHVN})$. AHVN is a combining architecture between $\mathrm{V} 2 \mathrm{~V}$ and $\mathrm{V} 2 \mathrm{R}$ networks. The interoperability between $\mathrm{V} 2 \mathrm{~V}$ and $\mathrm{V} 2 \mathrm{R}$ networks is considered. Then, we developed a Markov chain model in order to determine the MAC of AHVN based-collaborative. Both theoretical analysis and simulation experiments show that the proposed AHVN strengthen cooperative vehicular services and exploit physical roadway characteristics. Supporting a cooperative secure beaconing messages communication and developing an efficient packet scheduler in AHVN is our future work.

\section{ACKNOWLEDGMENT}

This project was supported by the Deanship of Scientific Research at Salman bin Abdulaziz University (SAU) under the research project \# 75/ت/1433

\section{REFERENCES}

[1] W. Chen, S. Cai, "Ad hoc peer to peer network architecture for vehicle safety communications," IEEE Communication. Magazine. Vol. 43, No. 4, April 2005, pp.100-107.

[2] I. Lequerica, P. M. Ruiz and V. Cabrera, "Improvement of Vehicular Communications by Using 3G Capabilities to Disseminate Control Information" IEEE Net, Vol.24, No.1, January/February 2010, pp.32-38.

[3] RITA: http://www.standards.its.dot.gov.

[4] W. Alasmary and W. Zhuang "The Mobility Impact in IEEE 802.11p Infrastructure less Vehicular Networks," Elsevier Ad Hoc Networks Journal, Vol.10, No. 2, March 2012, pp. 222-230.

[5] Ekram H., G. Chow, CM. Leung, R. Mcleod, J. Misic and O. Yang," Vehicular Telematics Over Heterogeneous Wireless networks: A Survey," Computer Communication, Vol. 33, 2010, pp.775793.

[6] A. V. Babu and V. K. Muhammed Ajeer, "Analytical Model for Connectivity of Vehicular Ad Hoc Networks in the Presence of Channel Randomness," Wiley, International Journal of Communication Systems, 2011, DOI: 10.1002/dac. 1379 .
[7] T. Taleb and A. Benslimmane, "Design Guidelines for a Networks Architecture Integrating V2V with 3G \& Beyond Networks," Proc. IEEE Globecom, 2010, pp.1-5.

[8] A. Benslimane, T. Taleb and R. Sivaraj "Dynamic Clustering-Based Adaptive Mobile Gateway Management in Integrated V2V-3G Heterogeneous Wireless Networks" IEEE Journal on Selected Areas in Comm, Vol. 29, No. 3, March 2011, pp. $559-570$.

[9] S. Chun Ng, W. Zhang, Y. Zhang, Y. Yang and G. Mao, "Analysis of Access and Connectivity Probabilities in Vehicular Relay Networks" IEEE Journal on Selected Areas in Communication,Vol.29, No.1,January 2011, pp.140-150.

[10] CarTalk, 2000, http://www.cartalk2000.net.

[11]FleetNet Program, 2001, http://www.fleetnet.de.

[12]T. Luan, X. Ling and X. (Sherman) Shen, "MAC performance Analysis for Vehicle to Infrastructure Comm," Proc. IEEE WCNC, 2010, pp.1-5.

[13]M. Sepulcre, J. Gozalvez, J. Harri and H. Hartenstein," Contextual Communications Congestion Control for Cooperative Vehicular Networks" IEEE Trans. on Wireless Comm, Vol. 10, No. 2, February 2011, pp.385-389.

[14] T. Zhou, H. Sharif, M. Hempel, P. Mahasukhon, W. Wang, and T. Ma "A Novel Adaptive Distributed Cooperative Relaying MAC Protocol for Vehicular Networks" IEEE Journal on Selected Areas in Comm, Vol. 29, No. 1, January 2011, pp.72-82.

[15] Sok-Ian Sou and Tonguz O.K." Enhancing V2V Connectivity through Roadside Units on Highways" IEEE Trans. Veh. Technology, Vol. 60, No. 8, January 2011, pp. 3586 - 3602.

[16] I. Zeadally, R. Hunt, Y. Chen, A. Irwin and A. Hassan, "Vehicular ad hoc networks (V2V): status, results and challenges" Springer Telecommunication Systems, Online First, 2012, pp.1-25.

[17]M. Schonhof, A. Kesting, M. Treiber, D. Helbinga,"Coupled Vehicle and Information Flows: Message Transport on a Dynamic Vehicle Network”, Elsevier, Physica A, vol. 363, no.1, April 2006, pp.73-81.

[18] ANSI-IEEE 802.11 Standard: "Wireless lan medium access control (MAC) and physical layer (PHY) specifications 802.11," 1999.

[19] C. Wang, X. Cheng and D. Laurenson, "Vehicle-toVehicle Channel Modeling and Measurements: Recent Advances and Future Challenges," IEEE Comm. Magz., Nov. 2009, pp. 96 -102.

[20] T. Murray, M. Cojocari and $\mathrm{Fu}$ Huirong,"Measuring the performance of IEEE $802.11 \mathrm{p}$ using ns-2 simulator for vehicular networks" Proc. IEEE EIT, 2008, 498 - 503.

[21] Abbani, N., Jomaa, M., Tarhini, T., Artail, H., ElHajj, W., "Managing Social Networks in vehicular networks using trust rules", IEEE Symposium on 
Wireless Technology and Applications (ISWTA), p.p: 168-173, Sept. 2011.

[22] Ridong Fei, Kun Yang, Xueqi Cheng, "A cooperative social and vehicular network and its dynamic bandwidth allocation algorithms", IEEE Conference on Computer Communications Workshops (INFOCOM WKSHPS), p.p.: 63-67, April 2011.

[23] I. Lequerica, M. García Longaron, P.M. Ruiz, "Drive and share: efficient provisioning of social networks in vehicular scenarios", IEEE Communications Magazine, Vol: 48, Issue: 11, p.p: 90-97, Nov. 2010.

[24] A. Alganas, Lin Xiaodong, A. Grami, "EVSE: An Efficient Vehicle Social Evaluation Scheme with Location Privacy Preservation for Vehicular Communications", IEEE International Conference on Communications (ICC), p.p.:1-5, June 2011.

\section{Authors Biography}

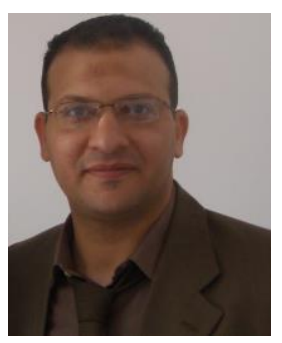

Saied M. Abd El-atty received the B.S. and M.S. degrees from Menoufia University, Faculty of Electronic Engineering, in 1995 and 2001, all in Electronics \& Communications Engineering respectively and $\mathrm{PhD}$ degree in Wireless Communication
Networks from University of Aegean (UOA) at the Information and Communication Systems Engineering Department, Greece, Samos in 2008. He is a member of the faculty members in the department of Electronics and Electrical Communication at Faculty of Electronic Engineering, Menouf, Egypt. Currently, he is working as assistant professor in Salman Bin Abulaziz University, KSA. He is the head of computer science and information department in Science College. Dr. Saied's current research interests include design, analysis, and optimization of wireless mobile communication networks and vehicular networks as well as cognitive radio systems. Majoring in Cross layer schemes, Handover, Radio Resource Management, Teletraffic, Vehicular Networks, Scheduling, small cell technology.

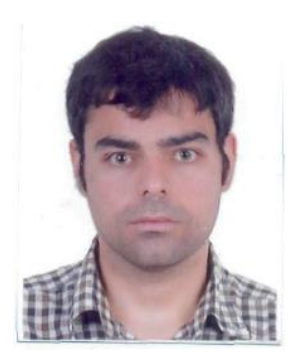

Konstantinos Lizos received his Engineering degree in 2003 and his M.S Degree in 2005, both with merit, from the Engineering Information \& Communication Systems Department, Samos, Greece at the University of Aegean (UOA). Since 2006, upon completing his military obligations, has joined the Hellenic Ministry of Foreign Affairs. He is currently associated with the $\mathrm{UiO}$ University in Norway as an active researcher in the fields of wireless networking. His current research interests include queue theory, mobile and vehicular communication networks, algorithms, signal and systems.

How to cite this paper: Saied M. Abd El-atty, Konstantinos Lizos,"Interoperability Framework for Vehicular Connectivity in Advanced Heterogeneous Vehicular Network", IJCNIS, vol.6, no.4, pp.1-12, 2014. DOI: 10.5815/ijcnis.2014.04.01 